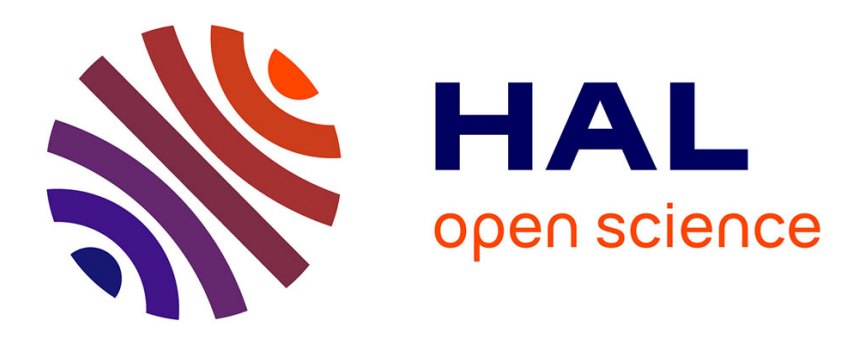

\title{
Superconductivity at the LaAlO3/SrTiO3interface
}

S. Gariglio, N. Reyren, A. D. Caviglia, J.-M. Triscone

\section{To cite this version:}

S. Gariglio, N. Reyren, A. D. Caviglia, J.-M. Triscone. Superconductivity at the LaAlO3/SrTiO3interface. Journal of Physics: Condensed Matter, 2009, 21 (16), pp.164213. 10.1088/0953-8984/21/16/164213 . hal-01591058

\section{HAL Id: hal-01591058 \\ https://hal.science/hal-01591058}

Submitted on 20 Sep 2017

HAL is a multi-disciplinary open access archive for the deposit and dissemination of scientific research documents, whether they are published or not. The documents may come from teaching and research institutions in France or abroad, or from public or private research centers.
L'archive ouverte pluridisciplinaire HAL, est destinée au dépôt et à la diffusion de documents scientifiques de niveau recherche, publiés ou non, émanant des établissements d'enseignement et de recherche français ou étrangers, des laboratoires publics ou privés. 


\title{
Superconductivity at the $\mathrm{LaAlO}_{3} / \mathrm{SrTiO}_{3}$ interface
}

\author{
S Gariglio, N Reyren, A D Caviglia and J-M Triscone \\ DPMC, University of Geneva, 24 Quai E.-Ansermet 1211 Genève 4, Switzerland \\ E-mail: stefano.gariglio@unige.ch
}

\begin{abstract}
We report on the structural characterization of $\mathrm{LaAlO}_{3} / \mathrm{SrTiO}_{3}$ interfaces and on their transport properties. $\mathrm{LaAlO}_{3}$ films were prepared using pulsed laser deposition onto $\mathrm{TiO}_{2}$ terminated (001) $\mathrm{SrTiO}_{3}$ substrates inducing a metallic conduction at the interface. Resistance and Hall effect measurements reveal a sheet carrier density between 0.4 and $1.2 \cdot 10^{14}$ electrons $/ \mathrm{cm}^{2}$ at room temperature and mobility of $\sim 300 \mathrm{~cm}^{2} \mathrm{~V}^{-1} \mathrm{~s}^{-1}$ at low temperatures. A transition to a superconducting state is observed at a temperature of $\sim 200 \mathrm{mK}$. The superconducting characteristics display signatures of $2 \mathrm{D}$ superconductivity.
\end{abstract}

\section{Introduction}

Ohtomo and Hwang discovered in 2004 that metallic conduction occurs at the interface between two insulating materials, $\mathrm{LaAlO}_{3}(\mathrm{LAO})$ and $\mathrm{SrTiO}_{3}$ (STO) [1]. This discovery has generated a lot of interest and motivated extensive theoretical and experimental work aimed at investigating the physics of complex oxide interfaces. In such systems, novel electronic properties have been predicted [2] and recently observed [3,4]. In the specific case of the LAO/STO interface, magnetic effects [5], superconductivity [6] and a rich electronic phase diagram [7] have been reported. The mechanism leading to the formation of a mobile electron gas at the LAO/STO interface remains today a highly debated issue. A possible explanation is the so-called 'polar catastrophe' scenario. According to this interpretation, the origin of this phenomenon is linked to a polar discontinuity occurring at the LAO/STO interface between the polar (001) LAO atomic planes and non-polar (001) STO planes [8]. When the polar LAO atomic planes are stacked on top of each other, an electric potential develops as the film thickness increases. Above a critical thickness, an electronic reconstruction may take place leading to a charge transfer at the interface, reducing the electrostatic energy and creating a two dimensional electron gas. Experimental evidences supporting this scenario have been reported in ref. [9], where the electron gas and metallic conduction have only been observed for LAO films at least 4 unit cells (u.c.) thick. The confinement of the electron gas has been estimated from analyses of the superconducting properties [10] and by room temperature conductive atomic force measurements [11]. These experiments lead to an estimate of the thickness of the electron gas of a few nanometers. In this short paper we report on the structural characterization of $\mathrm{LAO} / \mathrm{STO}$ structures and describe their transport properties in the normal and superconducting states. We analyze the superconducting behavior in the framework of the Berezinskii-KosterlitzThouless (BKT) theory of 2D superconductivity. 


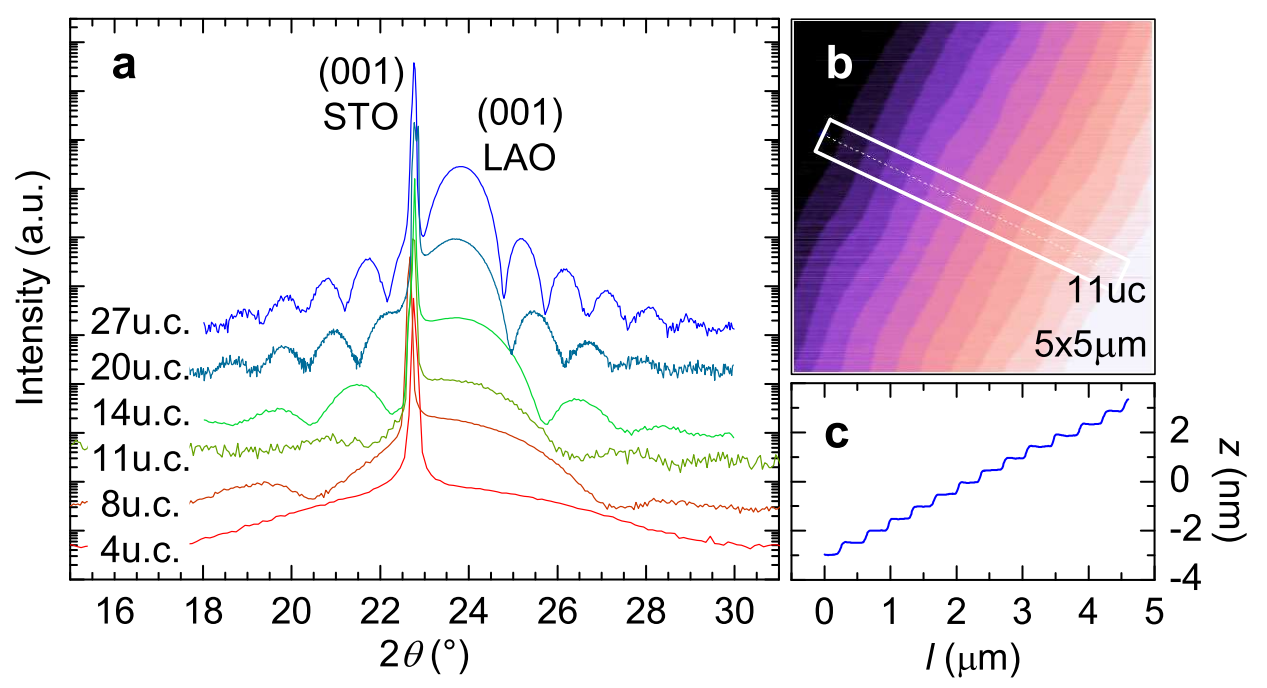

Figure 1. Structural characterization of LAO thin films. (a) X-ray $\theta-2 \theta$ diffractograms around the (001) LAO and STO reflections for LAO thin films with different thicknesses. (b) AFM topography performed on an 11 u.c. LAO thick film and (c) a height profile averaged over the width of the area delimited by the white lines. The step-and-terrace structure reflecting the miscut of the STO substrate is clearly visible.

\section{Experimental details}

Conducting interfaces were prepared depositing more than 3 u.c. of LAO on top of (001) $\mathrm{SrTiO}_{3}$ single crystals with $\mathrm{TiO}_{2}$-terminated surfaces. The films were grown by pulsed laser deposition (PLD) at $800^{\circ} \mathrm{C}$ in $1 \cdot 10^{-4}$ mbar of $\mathrm{O}_{2}$ with a repetition rate of $1 \mathrm{~Hz}$. The fluence of each laser pulse was $0.6 \mathrm{~J} / \mathrm{cm}^{2}$. After the LAO deposition, each sample was annealed in $200 \mathrm{mbar}$ of $\mathrm{O}_{2}$ at about $600^{\circ} \mathrm{C}$ for one hour and cooled down to room temperature in the same $\mathrm{O}_{2}$ background pressure. The growth was monitored in situ using reflection high energy electron diffraction (RHEED) which allows a control of the thickness with sub u.c. precision [12]. Samples with different thicknesses of LAO were systematically characterized, analyzing the surface morphology by atomic force microscopy (AFM) and crystalline quality by X-ray diffraction (XRD). The samples were then patterned in a geometry suitable for 4 point transport measurements. The patterning technique is based on the fact that only regions covered by more than 3 u.c. are conducting [9]. The LAO thickness was thus reduced using Argon ion milling down to 2 u.c. in specific regions of the samples while protecting the transport channels with photoresist. Once the conducting channels were defined, the transport properties were investigated in a dilution cryostat.

\section{Results}

Figure 1a displays $\theta-2 \theta$ X-ray diffractograms close to the (001) LAO and STO reflections for samples with different LAO thicknesses. As can be seen in the Figure, the period of finite size Fresnel oscillations around the diffraction peak changes with the LAO film thickness and allows the thickness of the LAO layer to be determined. The thickness obtained from the XRD data analyses is found to agree within one unit cell with the thickness deduced from the RHEED intensity oscillations. Figure $1 \mathrm{~b}$ shows a typical AFM topography measured on an 11 u.c. LAO sample. We observe that the steps-and-terraces structure of the substrate is reproduced on the surface of the film. The step height is about $4 \AA$, corresponding to one u.c of LAO, as shown in Figure 1c. 

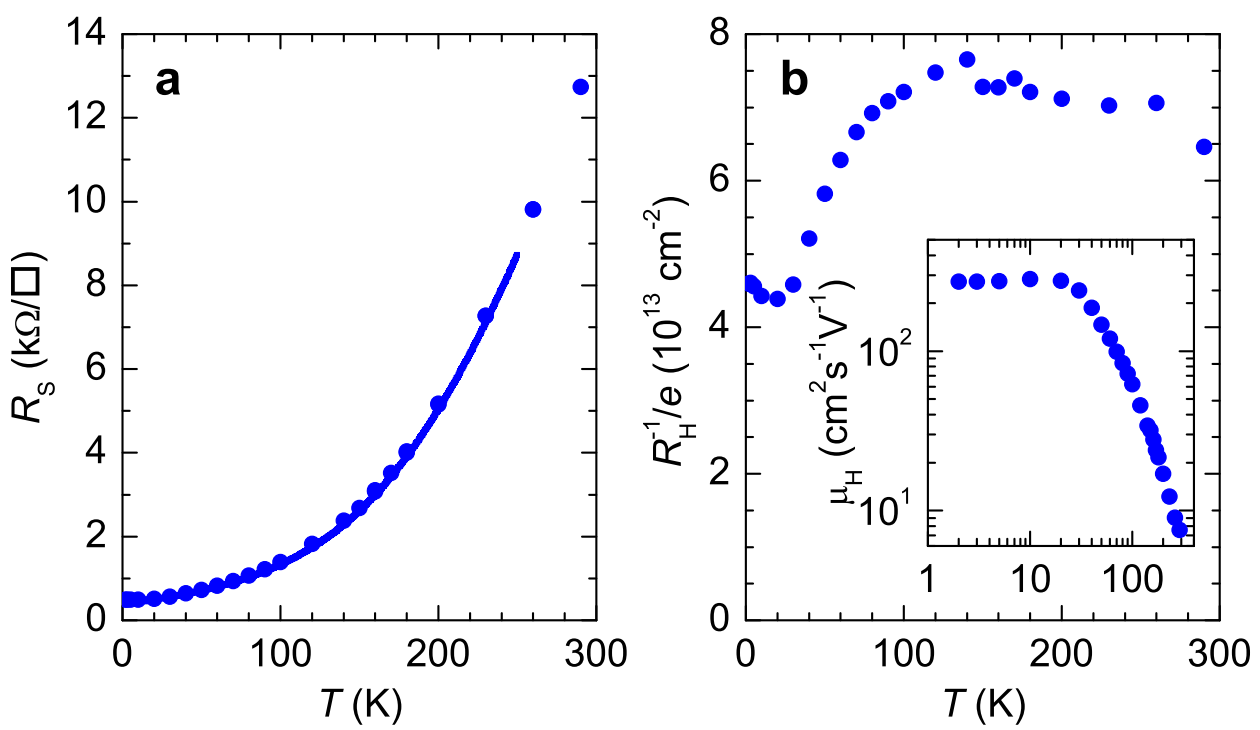

Figure 2. Transport properties in the normal state for a LAO/STO structure. (a) Sheet resistance as a function of temperature for a 4 u.c. thick LAO film grown onto a (001) STO substrate. The continuous line represents the resistance measurement during the cooling while the dots are measurements performed stabilizing the temperature during the heating cycle. (b) Inverse sheet Hall constant and mobility (inset) versus temperature for the same sample.

Reciprocal space maps (RSM) were performed to inspect the structural coherence of the LAO films grown atop the STO substrates. Measurements of RSM around the (103) diffraction peaks for film and substrate reveal that the in-plane lattice parameter of the LAO layer is strained to the one of STO. This demonstrates that LAO thin films grow pseudomorphically on STO substrates.

Transport measurements reveal that these heterostructures have a typical sheet resistance $R_{S}$ between 10 and $30 \mathrm{k} \Omega$ /square at room temperature. Most of the samples display a small photoelectric effect $(\Delta R / R<5 \%)$ under ambient light illumination. As shown on Figure 2a, resistance measurements indicate a metallic behavior down to $4 \mathrm{~K}$, with a residual resistivity ratio of the order of 25 . Figure $2 \mathrm{~b}$ displays the inverse sheet Hall constant as a function of temperature for the same sample. Considering a single band model, we estimate a sheet carrier density of the order of $7 \cdot 10^{13}$ electrons $/ \mathrm{cm}^{2}$ above $100 \mathrm{~K}$. From sample to sample, the sheet carrier density varies between 0.4 and $1.2 \cdot 10^{14}$ electrons $/ \mathrm{cm}^{2}$, without correlation with the thickness of the LAO layer. As can be noted in the Figure, below $100 \mathrm{~K}$, the inverse sheet Hall constant decreases as the temperature is reduced. Such a behavior has previously been observed in Nbdoped $\mathrm{SrTiO}_{3}$ thin films [13] and attributed to multiband conduction. Although this issue is still controversial, it is known that doped $\mathrm{SrTiO}_{3}$ possesses three degenerate bands for $\mathrm{Ti} 3 d$ electrons [14]. This degeneracy is lifted by the structural phase transition occurring at $\sim 105 \mathrm{~K}$. The inset of Figure $2 \mathrm{~b}$ shows the mobility versus temperature which reaches a value of $\sim 300 \mathrm{~cm}^{2} / \mathrm{Vs}$ at low temperatures.

Measurements of the resistance at low temperatures reveal that this electron gas condenses into a superconducting state. Figure 3a shows the sheet resistance versus temperature for a 4 u.c. thick LAO film. As can be seen, a superconducting transition occurs at a temperature of $\sim 220 \mathrm{mK}$. From magnetotransport measurements with the magnetic field applied perpendicular to the interface, we have estimated $H_{c 2}(0 \mathrm{~K})$ to be $\sim 90 \mathrm{mT}[6]$ which leads to a superconducting coherence length $\xi(0)$ of about $60 \mathrm{~nm}$. As the thickness of the electron gas is below $10 \mathrm{~nm}[6,11]$, 

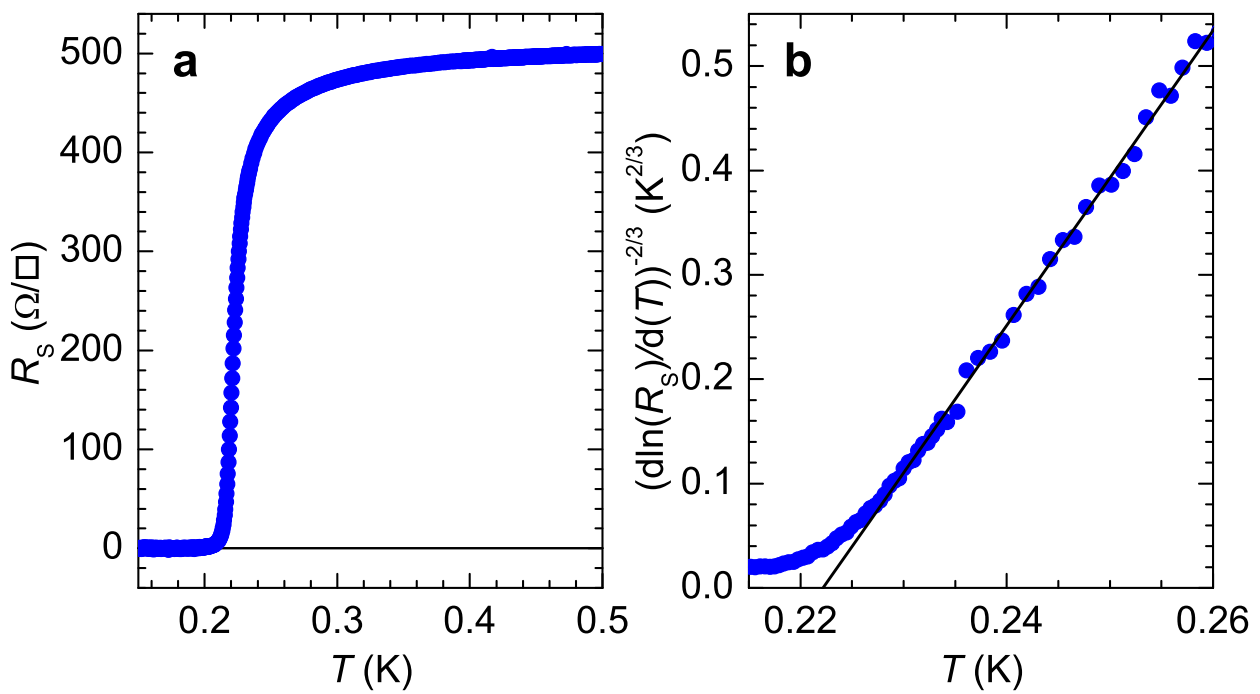

Figure 3. Transport properties at low temperatures.(a) Sheet resistance versus temperature for a 4 u.c. sample. (b) $\left(d \ln R_{S} / d T\right)^{-2 / 3}$ versus temperature revealing the linear behavior expected for a BKT transition: the extrapolation of the linear part to zero resistance leads to an estimate for $T_{B K T}$ of about $222 \mathrm{mK}$.

we expect the system to behave as a 2D superconductor possibly with footprints of a BerezinskiiKosterlitz-Thouless (BKT) transition $[15,16]$.

In the BKT scenario, the resistance as a function of temperature is expected to follow the relation: $R=R_{\circ} \cdot \exp \left[-b\left(T / T_{B K T}-1\right)^{-1 / 2}\right]$, where $T_{B K T}$ is the BKT transition temperature and $b$ a material dependent parameter. To estimate $T_{B K T}$ and $b$ we rewrite the expression as $(d \ln R / d T)^{-2 / 3}=(2 / b)^{2 / 3}\left(T-T_{B K T}\right)$. This form has the advantage that $b$ and $T_{B K T}$ can be estimated from the characteristic linear behavior above $T_{B K T}$, without having to determine $R_{\circ}$. This analysis is shown in Figure $3 \mathrm{~b}$, where we notice that the resistance measurements are consistent with the BKT behavior. The rounding of the transition at the lowest temperatures is attributed to a size effect. The linear extrapolation to $R=0$ leads to $T_{B K T}=222 \mathrm{mK}$.

\section{Conclusion}

We have discussed the growth and characterization of LAO films grown onto (001) STO $\mathrm{TiO}_{2}{ }^{-}$ terminated substrates. With the preparation conditions described above, we observe that only structures with a LAO thickness greater than 3 u.c. are conducting. We have shown that the electron gas present at these LAO/STO interfaces condenses into a superconducting state displaying properties expected for a $2 \mathrm{D}$ superconductor.

\section{Acknowledgments}

We thank Anna-Sabina Ruetschi, Didier Jaccard, Jochen Mannhart, Toni Schneider and Marc Gabay for useful discussions and Marco Lopes for his technical assistance. We acknowledge financial support by the Swiss National Science Foundation through the National Center of Competence in Research 'Materials with Novel Electronic Properties' MaNEP and Division II, by the European Union through the project 'Nanoxide' and by the European Science Foundation through the program 'Thin Films for Novel Oxide Devices'. 


\section{References}

[1] Ohtomo A and Hwang H Y 2004 Nature 427423

[2] Okamoto S and Millis A 2004 Nature 428 630-633

[3] Hwang H Y 2006 Mater. Res. Soc. Bull. 31 28-35

[4] Bousquet E, Dawber M, Stucki N, Lichtensteiger C, Hermet P, Gariglio S, Triscone J M and Ghosez P 2008 Nature 452 732-736

[5] Brinkman A, Huijben M, van Zalk M, Huijben J, Zeitler U, Maan J C, van der Wiel W G, Rijnders G, Blank D H and Hilgenkamp H 2007 Nat. Mater. 6 493-496

[6] Reyren N, Thiel S, Caviglia A D, Kourkoutis L F, Hammerl G, Richter C, Schneider C W, Kopp T, Retschi A S, Jaccard D, Gabay M, Muller D A, Triscone J M and Mannhart J 2007 Science 317 1196-1199

[7] Caviglia A D, Gariglio S, Reyren N, Jaccard D, Schneider T, Gabay M, Thiel S, Hammerl G, Mannhart J and Triscone J M 2008 Nature

[8] Nakagawa N, Hwang H Y and Muller D 2006 Nat. Mater. 5 204-209

[9] Thiel S, Hammerl G, Schmehl A, Schneider C W and Mannhart J 2006 Science $3131942-1945$

[10] Reyren N 2009 In preparation

[11] Basletic M, Maurice J L, Carrétéro C, Herranz G, Copie O, Bibes M, Jacquet E, Bouzehouane K, Fusil S and Barthélémy A 2008 Nat. Mater. 7 621-625

[12] Rijnders G, Koster G, Blank D H A and Rogalla H 1997 Appl. Phys. Lett. 70 1888-1890

[13] Takahashi K S, Gabay M, Jaccard D, Shibuya K, Ohnishi T, Lippmaa M and Triscone J M 2006 Nature 441 195-198

[14] Mattheiss L F 1972 Phys. Rev. B 64740

[15] Berezinskii V L 1972 Sov. Phys. JETP 34610

[16] Kosterlitz J M and Thouless D J 1972 J. Phys. C 5 L124 